\title{
Cascade Observers for Nonlinear Systems and Application to Nonlinear Output Feedback Control*
}

\author{
Dong Hun $\mathrm{KIM}^{* *}$, Hua WANG*** and Eung-Seok $\mathrm{KIM}^{* * * *}$
}

\begin{abstract}
In this paper, we present a new observer, namely a cascade observer, for a general class of nonlinear SISO systems. Inspired by but different from a high-gain observer, the cascade observer features a cascade structure and adaptive observer gains. In doing so the cascade observer attempts to overcome some of the typical problems that may pose to a high-gain observer. As in the case of a high-gain observer, the cascade observer structure is simple and universal in the sense that it is independent of the system dynamics and parameters. It is shown that the estimation errors converge to zero and all the internal variables are globally uniformly bounded. Based on the cascade observer, an adaptive output feedback controller is designed for a class of SISO nonlinear systems represented globally by a $n$ th-order differential equation. The output feedback controller has the same structure as a state feedback controller. Globally uniformly ultimate boundedness of tracking errors is achieved for the nonlinear systems with unknown parameters.
\end{abstract}

\section{Key Words: Cascade Observer, High-Gain Observer, Output Feedback Control, Adaptive} Law

\section{Introduction}

Observer design for nonlinear systems has been a long standing challenging problem in the field of systems and control. Typical observer designs such as Luenberger observer $^{(1)}$ and sliding observers ${ }^{(2)}{ }^{(5)}$ require the knowledge of system dynamics. In contrast, a high-gain observer $^{(6)-(8)}$ has been developed for a class of SISO nonlinear systems that are transformable into a nominal triangular form and its structure is independent of system dynamics and parameters. The difficulty arising in practical applications, however, is the determination of an appropriate value for the observer gain. By choosing the observer gain large enough (therefore the term "high-gain"), the observer error can be made arbitrarily small. But for high values, initial peaking (called 'peaking phenomenon') is generated, i.e. large mismatched values between real and

* Received 27th June, 2005 (No. 05-5065)

** Division of Electronics and Electrical Engineering, Kyungnam University, Masan, Kyungnam 631-701, Korea. E-mail: dhkim@kyungnam.ac.kr

*** Department of Aerospace and Mechanical Engineering, Boston University, Boston, MA 02215, USA.

E-mail: wangh@bu.edu

**** Department of Electrical Engineering, Halla University, Wonju, Kangwon-Do 220-712, Korea.

E-mail: eskim@hit.halla.ac.kr estimated values for the short initial period ${ }^{(28)}$. For an illustration of the peaking phenomenon in high-gain observers, see Ref. (35). For values too low, desired bounds on the observer error can not be achieved. The high-gain observer induces a time scale separation between the considered system and the estimate error dynamics; hence, the singular perturbation techniques ${ }^{(9)}$ have been used in the stability analysis of the error dynamics ${ }^{(27)}$. One way to remedy the velocity or acceleration feedback problem is to determine a velocity or acceleration signal by firstorder or second-order numerical differentiation of the accurate position signal. The simplicity of this technique makes it particularly useful from an implementation point of view. However, for low and high velocities especially, such a simple approximation of the velocity signal may be inadequate ${ }^{(30),(31)}$. Moreover, the quantization effect that inherently goes along with this approach may produce undesired oscillations in the robot joint response, or even cause it to become unstable ${ }^{(32)}$. In addition, there is no theoretical justification for this ad hoc solution.

Inspired by the high-gain observer design, in this paper, we propose a new observer design with a cascade structure, referred as a cascade observer. The new cascade observer is applicable to the same class of SISO nonlinear systems as in the case of a high-gain observer. In addition to the cascade structure, the gains of a cascade observer consist of two type: fixed and adaptive ones. In doing so 
the cascade observer attempts to overcome some of the typical problems outlined above for a high-gain observer. The cascade observer structure is simple and universal in the sense that it is independent of the system dynamics and parameters. It is shown that the estimation errors converge to zero and all the internal variables are globally uniformly bounded.

Following the development of cascade observers, we address the problem of adaptive output feedback for a class of SISO nonlinear systems based on the cascade observers. In this regard, high-gain observers based output feedback for nonlinear systems have been considered in a number of studies ${ }^{(10)-(16)}$. The works of Refs. (10) and (16) require persistence of excitation for tracking error convergence. In all these schemes, the selection of an appropriate observer gain could be a potential problem in practical implementations. In this paper, we formulate and solve a nonlinear output feedback problem using the proposed cascade observer for a class of SISO nonlinear system represented globally by a $n$ th-order differential equation. The output feedback controller has the same structure as a state feedback controller. Globally uniformly ultimate boundedness of tracking errors is achieved for the nonlinear systems with unknown parameters.

This paper is organized as follows. In section 2, we present the problem statement and recall the structure of a high-gain observer. In section 3, we introduce the new cascade observer and present its stability analysis. Examples are given to illustrate the theory and compare with a high-gain observer. In section 4 , the proposed observer is used for the design of adaptive output feedback controller for a class of SISO nonlinear systems with unknown constant parameters. In section 5, concluding remarks are collected.

\section{Problem Statement and High-Gain Observer}

Consider analytic (for the sake of simplicity) systems of the form

$$
\Sigma:\left\{\begin{array}{l}
\dot{x}=f(x)+g(x) u \\
y=h(x)
\end{array}\right.
$$

where $x \in \in R^{n}$ is the state, $u \subset \in R$ is the control input, and $y \in \in R$ is the measured output. Moreover there is a "physical subset" $\Omega \subset \in R^{n}$ under consideration, on which we are interested in the observation problem. In most practical cases, $\Omega$ will be an open connected relatively compact subset of $\in R^{n}$, and in the ideal cases, $\Omega$ will be positivelyinvariant under the dynamics $(\Sigma)$.

Assume that $(\Sigma)$ is observable on $\Omega$ and that $u \equiv 0$ is a universal input ${ }^{(33)}$. The fact that $(\Sigma)$ is observable is equivalent to the requirement that the set of functions, called the "observation space" of $(\Sigma), \Theta(\Sigma)=\left\{h, L_{f} h, \cdots, L_{f}^{i} h, \cdots \mid i \geq\right.$ $0\}$ separates the point on $\Omega$, i.e. $\forall x_{1}, x_{2} \in \Omega, \exists i$, s.t. $L_{f}^{i} h\left(x_{1}\right) \neq L_{f}^{i} h\left(x_{2}\right)$ ( $L$ denotes the Lie derivative operator).
This is due to the formula $y(t)=\sum_{k=0}^{+\infty} L_{f}^{k} h\left(x_{0}\right) t^{k} / k !$. It is easily seen that when $(\Sigma)$ is observable, the map

$$
F_{\Sigma}:\left\{x \rightarrow\left(\begin{array}{c}
h(x) \\
L_{f} h(x) \\
\vdots \\
L_{f}^{n-1} h(x)
\end{array}\right)\right.
$$

is almost everywhere regular (see, for instance, Ref. (34)).

The following assumptions will be made throughout the paper.

Assumption $1 F_{\Sigma}$ is a diffeomorphism from $\Omega$ onto $F_{\Sigma}(\Omega)$.

From this assumption it follows that, on $(\Omega)$, the global coordinate system defined by $F_{\Sigma}$ can be written as

$$
\left(\Sigma^{\prime}\right):\left\{\dot{x}=\left[\begin{array}{c}
\dot{x}_{1} \\
\dot{x}_{2} \\
\vdots \\
\dot{x}_{n-1} \\
\dot{x}_{n}
\end{array}\right]=\left[\begin{array}{c}
x_{2} \\
x_{3} \\
\vdots \\
x_{n} \\
\phi(x, u)
\end{array}\right] \quad, \quad y=x_{1} .\right.
$$

Assumption $2 \phi$ is globally Lipschitz with respect to $x$ and uniformly respect to $u$.

Definition: When Assumptions 1 and 2 hold, we say that $(\Sigma)$ is uniformly observable on $\in R^{n}$.

Uniform observability clearly means that the initial state can be reconstructed on the basis of the data formed by the output and its $(n-1)$ first derivatives.

One approach to observe such systems $\left(\Sigma^{\prime}\right)$ is through the use of a high-gain observer ${ }^{(29)}$. The structure of a high-gain observer is a simple chain of integrators, each corrected by the injection of the output error $\left(y-\hat{x}_{1}\right)$ multiplied by a factor depending on the constant observer gain $\frac{1}{\epsilon}$ :

$$
\begin{aligned}
& \dot{\hat{x}}_{1}=\hat{x}_{2}+l_{1}\left(y-\hat{x}_{1}\right) / \epsilon \\
& \dot{\hat{x}}_{i}=\hat{x}_{i+1}+l_{i}\left(y-\hat{x}_{1}\right) / \epsilon^{i}, i=2, \cdots, n-1 \\
& \dot{\hat{x}}_{n}=l_{n}\left(y-\hat{x}_{1}\right) / \epsilon^{n} \\
& \hat{y}=\hat{x}_{1}
\end{aligned}
$$

where $\hat{x}=\left[\hat{x}_{1}, \cdots, \hat{x}_{n}\right]^{T}$ denotes the estimate of the states $x$ and $\hat{y}$ denotes the estimate of the system output.

In contrast to a classical Luenberger observer ${ }^{(1)}$, a high-gain observer does not consist of a replica of the system $\left(\Sigma^{\prime}\right)$ plus correction terms as the nonlinearity $\phi(x, u)$ is not utilized. The observer error is denoted by $e(t)$ with $e(t)=\left[e_{1}(t), \cdots, e_{n}(t)\right]^{T}$ with $e_{i}(t)=x_{i}(t)-\hat{x}_{i}(t)$.

Assumption 3 The nonlinearity $\phi$ in $\left(\Sigma^{\prime}\right)$ is bounded, i.e.

$$
\|\phi(x, u)\| \leq \mu, \quad \forall x, u .
$$

If the coefficients $l_{1}, \cdots, l_{n}$ are such that $s^{n}+\sum_{j=1}^{n} l_{j} s^{n-j}$ is a Hurwitz polynomial with distinct roots, then for all $d>0$ 
and all times $\bar{t}>0$ there exists a finite observer gain $\frac{\overline{1}}{\epsilon}$ such that for all constant $\frac{1}{\epsilon} \geq \frac{\overline{1}}{\epsilon}$ the observer error satisfies:

$$
\|e(t)\| \leq d, \quad \forall t \geq \bar{t} .
$$

This means that by an appropriate choice of the observer gain $\frac{1}{\epsilon}$ the observer error $e$ can be made arbitrarily small. However, the determination of an appropriate observer gain can be quite difficult in practical applications of highgain observers. In order to make the observer error small, high gains are preferred. But for high values, the peaking phenomenon can be undesirable ${ }^{(28)}$. For values too low, desired bounds on the observer error can not be achieved. In the following, we propose a cascade observer design that attempts to overcome some of the problems associated with high-gain observers.

\section{Cascade Observer}

\subsection{Observer design}

A basic design objective of the proposed cascade observer is to guarantee the convergence of observer errors in a 'cascade' fashion, that is, $\hat{x}_{1}=x_{1} \rightarrow \hat{x}_{2}=\dot{\hat{x}}_{1} \rightarrow \hat{x}_{2}=\dot{\hat{x}}_{1}=$ $\dot{x}_{1} \rightarrow \hat{x}_{3}=\dot{\hat{x}}_{2} \rightarrow \hat{x}_{3}=\dot{\hat{x}}_{2}=\ddot{x}_{1}, \cdots, \hat{x}_{n+1}=\dot{\hat{x}}_{n} \rightarrow \hat{x}_{n+1}=\dot{\hat{x}}_{n}=x_{1}^{(n)}$. Based on this concept, a cascade observer is designed as

$$
\begin{aligned}
& \dot{\hat{x}}_{1}=\hat{x}_{2}+l_{1}\left(y-\hat{x}_{1}\right)+\hat{\rho}_{1} \operatorname{sgn}\left(y-\hat{x}_{1}\right) \\
& \dot{\hat{x}}_{i}=\hat{x}_{i+1}+l_{i}\left(\dot{\hat{x}}_{i-1}-\hat{x}_{i}\right)+\hat{\rho}_{i} \operatorname{sgn}\left(\dot{\hat{x}}_{i-1}-\hat{x}_{i}\right), i=2, \cdots, n \\
& \dot{\hat{x}}_{n+1}=l_{n+1}\left(\dot{\hat{x}}_{n}-\hat{x}_{n+1}\right)+\hat{\rho}_{n+1} \operatorname{sgn}\left(\dot{\hat{x}}_{n}-\hat{x}_{n+1}\right) \\
& \hat{y}=\hat{x}_{1}
\end{aligned}
$$

where we utilize the condition such that $\left|\hat{x}_{i+1}-\ddot{\hat{x}}_{i-1}\right| \leq \rho_{i}$, $i=1, \cdots, n . i$ is a step number.

$$
\operatorname{sgn}(a):=\left(\begin{array}{rll}
1 & \text { if } & a>0 \\
0 & \text { if } & a=0 \\
-1 & \text { if } & a<0
\end{array}\right)
$$

Note that $\rho_{i}, i=1, \cdots, n$ and $\rho_{n+1}$ are upper bound values and constants. And this will be verified through Theorem 1. It is also shown in Theorem 1 that the $\rho_{i}$ 's exist and are bounded. $\hat{\rho}_{i}$ denotes the estimate of $\rho_{i}$, and $l_{i}$ is a positive constant. When $l_{i}$ s are chosen as commonly used, $l_{i}$ will be larger than $l_{i+1}$ because the estimation of the former step contributes more than the estimation of the latter one in terms of the cascade construction. Such a gain selection requires a smaller gain value as the state order increases, unlike the high-gain observer demands a higher gain value as the state order increases. Figure 1 shows the structure of the proposed observer. Each step is connected in a cascade way. The existence of solutions to the proposed observers is based on the Fillipov's solutions ${ }^{(36)}$ to differential equations, which have discontinuous right-hand sides.

Notation. For convenience, $\dot{\hat{x}}_{0}$, in this note, refers to $y$.

Adaptive law of $\hat{\rho}_{i}$ in (4) is designed as

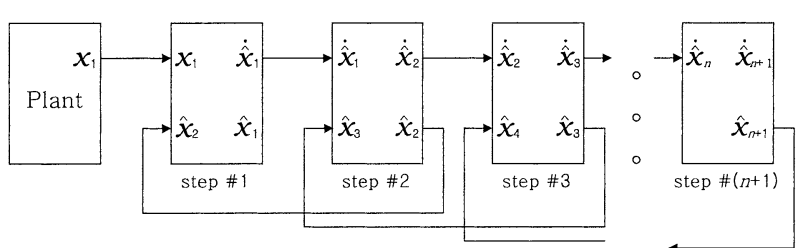

Fig. 1 The structure of cascade observer

$$
\begin{aligned}
\dot{\hat{\rho}}_{i} & =\gamma_{i}\left|\dot{\hat{x}}_{i-1}-\hat{x}_{i}\right| \quad \text { if } \quad \hat{\rho}_{i} \leq \bar{\rho}_{i} \quad i=1, \cdots, n+1 \\
& =\gamma_{i}\left[1+\frac{\bar{\rho}_{i}-\hat{\rho}_{i}}{\delta_{i}}\right]\left|\dot{\hat{x}}_{i-1}-\hat{x}_{i}\right| \quad \text { otherwise }
\end{aligned}
$$

where $\delta_{i}$ is a positive constant that plays the role to reduce the adaptation gain in the case of $\hat{\rho}_{i}>\bar{\rho}_{i}$ and prevent $\hat{\rho}_{i}$ from being divergent. $\bar{\rho}_{i}$ can be set to any positive value but should be chosen judiciously as they affect the transient response of the cascade observer. Equation (5) is the updating law to ensure that $\hat{\rho}_{i}$ is bounded.

Remark 1 The upper bounds of $\left|\hat{x}_{i+1}-\ddot{\hat{x}}_{i-1}\right|$ and $\left|\ddot{\hat{x}}_{n}\right|$, i.e. the $\rho_{i}$ 's, can be obtained under certain circumstances (please note that the $\rho_{i}$ 's exist and are bounded as shown in Theorem 1). For example, in the case of vehicle dynamics, the maximum velocity and acceleration values are known from performance specifications. However, to determine the values of these upper bounds a priori can be troublesome or may be difficult under certain circumstances. Therefore it is often more desirable to estimate them using adaptation laws. As in typical adaptation schemes, it does not matter that the estimated values do not converge to the real ones. The estimated values serve to organize the cascade observer to guarantee stability in the sense of Theorem 1.

Theorem 1 For system $\left(\Sigma^{\prime}\right)$ satisfying assumption assumption 3, the proposed cascade observer guarantees asymptotic stability of the estimation error.

Proof: To examine the stability analysis of the proposed observer, we consider the Lyapunov function candidate

$$
V=\frac{1}{2} \sum_{i=1}^{n+1}\left(\dot{\hat{x}}_{i-1}-\hat{x}_{i}\right)^{2}+\frac{1}{2} \sum_{i=1}^{n+1} \frac{1}{\gamma_{i}} \tilde{\rho}_{i}^{2}
$$

where $\tilde{\rho}_{i}=\rho_{i}-\hat{\rho}_{i}$. Differentiating $V$ along the system trajectory gives

$$
\dot{V}=\sum_{i=1}^{n+1}\left(\ddot{\hat{x}}_{i-1}-\dot{\hat{x}}_{i}\right)\left(\dot{\hat{x}}_{i-1}-\hat{x}_{i}\right)+\sum_{i=1}^{n+1} \frac{1}{\gamma_{i}} \tilde{\rho}_{i} \dot{\tilde{\rho}}_{i}
$$

Using (4) in (7) yields

$$
\begin{aligned}
\dot{V}= & -\sum_{i=1}^{n+1}\left(\dot{\hat{x}}_{i-1}-\hat{x}_{i}\right)\left[\hat{x}_{i+1}+l_{i}\left(\dot{\hat{x}}_{i-1}-\hat{x}_{i}\right)+\hat{\rho}_{i} \operatorname{sgn}\left(\dot{\hat{x}}_{i-1}-\hat{x}_{i}\right)\right] \\
& +\sum_{i=1}^{n+1} \ddot{\hat{x}}_{i-1}\left(\dot{\hat{x}}_{i-1}-\hat{x}_{i}\right)+\sum_{i=1}^{n+1} \frac{1}{\gamma_{i}} \tilde{\rho}_{i} \dot{\tilde{\rho}}_{i} \\
= & -\sum_{i=1}^{n+1} l_{i}\left(\dot{\hat{x}}_{i-1}-\hat{x}_{i}\right)^{2}-\sum_{i=1}^{n+1}\left(\dot{\hat{x}}_{i-1}-\hat{x}_{i}\right) \\
& \times\left[\hat{x}_{i+1}-\ddot{\hat{x}}_{i-1}+\hat{\rho}_{i} \operatorname{sgn}\left(\dot{\hat{x}}_{i-1}-\hat{x}_{i}\right)\right]+\sum_{i=1}^{n+1} \frac{1}{\gamma_{i}} \tilde{\rho}_{i} \dot{\tilde{\rho}}_{i}
\end{aligned}
$$




$$
\begin{aligned}
\leq & -\sum_{i=1}^{n+1} l_{i}\left(\dot{\hat{x}}_{i-1}-\hat{x}_{i}\right)^{2}+\sum_{i=1}^{n+1}\left|\dot{\hat{x}}_{i-1}-\hat{x}_{i}\right|\left|\hat{x}_{i+1}-\ddot{\hat{x}}_{i-1}\right| \\
& -\sum_{i=1}^{n+1} \hat{\rho}_{i} \operatorname{sgn}\left(\dot{\hat{x}}_{i-1}-\hat{x}_{i}\right)\left(\dot{\hat{x}}_{i-1}-\hat{x}_{i}\right)+\sum_{i=1}^{n+1} \frac{1}{\gamma_{i}} \tilde{\rho}_{i} \dot{\tilde{\rho}}_{i} \\
\leq & -\sum_{i=1}^{n+1} l_{i}\left(\dot{\hat{x}}_{i-1}-\hat{x}_{i}\right)^{2}+\sum_{i=1}^{n+1} \rho_{i}\left|\dot{\hat{x}}_{i-1}-\hat{x}_{i}\right|-\sum_{i=1}^{n+1} \hat{\rho}_{i}\left|\dot{\hat{x}}_{i-1}-\hat{x}_{i}\right| \\
& +\sum_{i=1}^{n+1} \frac{1}{\gamma_{i}} \tilde{\rho}_{i} \dot{\tilde{\rho}}_{i} \\
= & -\sum_{i=1}^{n+1} l_{i}\left(\dot{\hat{x}}_{i-1}-\hat{x}_{i}\right)^{2}+\sum_{i=1}^{n+1} \tilde{\rho}_{i}\left|\dot{\hat{x}}_{i-1}-\hat{x}_{i}\right|-\sum_{i=1}^{n+1} \frac{1}{\gamma_{i}} \tilde{\rho}_{i} \dot{\hat{\rho}}_{i} .(8)
\end{aligned}
$$

Substituting (5) into (8) gives

$$
\dot{V} \leq-\sum_{i=1}^{n+1} l_{i}\left(\dot{\hat{x}}_{i-1}-\hat{x}_{i}\right)^{2} .
$$

From Eq. (9), we can recognize that $\left(\dot{\hat{x}}_{i-1}-\hat{x}_{i}\right)$ and $\tilde{\rho}_{i}$ are bounded. Also, $\hat{\rho}_{i}$ is bounded from Eq. (5). Thus, $\rho_{i}$ and $\left|\hat{x}_{i+1}-\ddot{\hat{x}}_{i-1}\right|$ are bounded. Integrating Eq. (9) gives

$$
\begin{gathered}
\int_{0}^{t} \sum_{i=1}^{t n+1} l_{i}\left(\dot{\hat{x}}_{i-1}(\tau)-\hat{x}_{i}(\tau)\right)^{2} d \tau \leq-V(t)+V(0) \leq V(0) \\
=\frac{1}{2} \sum_{i=1}^{n+1}\left(\dot{\hat{x}}_{i-1}(0)-\hat{x}_{i}(0)\right)^{2}+\sum_{i=1}^{n+1} \frac{1}{2 \gamma_{i}} \tilde{\rho}_{i}^{2}(0) .
\end{gathered}
$$

Thus, because $V(0)$ and $l_{i}$ are constants, $\sum_{i=1}^{n+1}\left(\dot{\hat{x}}_{i-1}-\hat{x}_{i}\right) \in L_{2}$. Since we have proven that $\rho_{i}, \hat{\rho}_{i},\left(\dot{\hat{x}}_{i-1}-\hat{x}_{i}\right)$ and $\left|\hat{x}_{i+1}-\ddot{\hat{x}}_{i-1}\right|$ are bounded, we have $\sum_{i=1}^{n+1} \frac{d}{d t}\left(\dot{\hat{x}}_{i-1}-\hat{x}_{i}\right) \in L_{\infty}$. Using the Barbalat's Lemma $^{(17)}, \lim _{t \rightarrow \infty} \sum_{i=1}^{n+1}\left(\dot{\hat{x}}_{i-1}(t)-\hat{x}_{i}(t)\right)=0$.

Remark 2 From Theorem 1, it follows that state estimation errors converge to 0 in a 'cascade' fashion, i.e., first $\hat{x}_{1}$ converges to $x_{1}, \hat{x}_{2}$ then converges to $\dot{\hat{x}}_{1}=\dot{x}_{1}$. It follows that $\hat{x}_{3}$ converges to $\dot{\hat{x}}_{2}=\ddot{x}_{1}$. Therefore $\hat{x}_{i}$ converges to $\dot{\hat{x}}_{i-1}=x_{1}^{(i-1)}$. Moreover, the succession of convergence implies that $\hat{x}_{i}$ are bounded. Therefore all the internal variables are globally uniformly bounded.

Theorem 2 (Stability without adaptation) For system $\left(\Sigma^{\prime}\right)$ satisfying assumption 1, the proposed cascade observer with $\gamma_{i}=0$ guarantees that the estimation errors is globally uniformly bounded.

Proof: Since $\gamma_{i}=0$, the parameter estimate $\hat{\rho}_{i}$ is constant. For the nonadaptive Lyapunov function candidate (6), we consider

$$
V_{c 1}=\frac{1}{2} \sum_{i=1}^{n+1}\left(\dot{\hat{x}}_{i-1}-\hat{x}_{i}\right)^{2} .
$$

From Eq. (8) we have

$$
\dot{V}_{c 1}=-\sum_{i=1}^{n+1} l_{i}\left(\dot{\hat{x}}_{i-1}-\hat{x}_{i}\right)^{2}+\sum_{i=1}^{n+1} \tilde{\rho}_{i}\left|\dot{\hat{x}}_{i-1}-\hat{x}_{i}\right| .
$$

Let us denote $\chi=2 V_{c 1}=\sum_{i=1}^{n+1}\left(\dot{\hat{x}}_{i-1}-\hat{x}_{i}\right)^{2}, \quad l_{0}=(n+$ 1) $\min _{1 \leq i \leq n+1} l_{i}$ and $\tilde{\rho}_{0}=(n+1) \max _{1 \leq i \leq n+1} \tilde{\rho}_{i}$. Then if we use $\sum_{i=1}^{n+1}\left|\dot{\hat{x}}_{i-1}-\hat{x}_{i}\right| \leq \sqrt{n+1}\left\{\sum_{i=1}^{n+1}\left(\dot{\hat{x}}_{i-1}-\hat{x}_{i}\right)^{2}\right\}^{\frac{1}{2}}=\sqrt{n+1} \chi^{\frac{1}{2}}$ by Schwarz Inequality ${ }^{(25)}$, we have

$$
\frac{d}{d t} \chi \leq-2 l_{0} \chi+2 \sqrt{n+1} \tilde{\rho}_{0} \chi^{\frac{1}{2}} .
$$

This type of equation is solved via a substitution. Indeed, let $\eta=\chi^{\frac{1}{2}}$, then

$$
\frac{d \eta}{d t}=\frac{1}{2} \chi^{-\frac{1}{2}} \frac{d \chi}{d t} .
$$

Rewriting (13) gives

$$
\chi^{-\frac{1}{2}} \frac{d \chi}{d t}=2 \sqrt{n+1} \tilde{\rho}_{0}-2 l_{0} \chi^{\frac{1}{2}}=2 \sqrt{n+1} \tilde{\rho}_{0}-2 l_{0} \eta .
$$

Plugging (15) into (14) gives

$$
\dot{\eta}+l_{0} \eta=\sqrt{n+1} \tilde{\rho}_{0} .
$$

Solving the linear equation to find $\eta$ gives

$$
\begin{aligned}
\eta(t) & =\sqrt{n+1} \frac{\tilde{\rho}_{0}}{l_{0}}\left(1-e^{-l_{0} t}\right)+\eta(0) e^{-l_{0} t} \\
& =\sqrt{n+1} \frac{\tilde{\rho}_{0}}{l_{0}}+e^{-l_{0} t}\left(\eta(0)-\sqrt{n+1} \frac{\tilde{\rho}_{0}}{l_{0}}\right) .
\end{aligned}
$$

Going back to $\chi$ through the substitution $\chi=\eta^{2}$ gives

$$
\begin{aligned}
\chi(t)= & \left\{\sqrt{n+1} \frac{\tilde{\rho}_{0}}{l_{0}}+e^{-l_{0} t}\left(\chi(0)^{\frac{1}{2}}-\sqrt{n+1} \frac{\tilde{\rho}_{0}}{l_{0}}\right)\right\}^{2} \\
\leq & (n+1)\left(\frac{\tilde{\rho}_{0}}{l_{0}}\right)^{2}+\left\{2 \sqrt{n+1} \frac{\tilde{\rho}_{0}}{l_{0}}\left(\chi(0)^{\frac{1}{2}}-\sqrt{n+1} \frac{\tilde{\rho}_{0}}{l_{0}}\right)\right. \\
& \left.+\left(\chi(0)^{\frac{1}{2}}-\sqrt{n+1} \frac{\tilde{\rho}_{0}}{l_{0}}\right)^{2}\right\} e^{-l_{0} t} .
\end{aligned}
$$

This proves that the solution $\chi$ is globally uniformly bounded without adaptation, i.e., $\chi(t) \rightarrow(n+1)\left(\frac{\tilde{\rho}_{0}}{l_{0}}\right)^{2}$ as $t \rightarrow \infty$.

Two case studies will be presented to illustrate the performance of the proposed cascade observer. In the first case, the estimation of a signal $y=10 \sin t$ is carried out. The second case study addresses the state estimation of an underwater vehicle. In both cases the results of the proposed cascade observer are compared with those of the high-gain observer.

\subsection{Example 1}

We illustrate the results of the proposed observer via comparison with a high-gain observer. Figure 2 shows the results for estimating the derivatives of $x_{1}=10 \sin t$. Note that this estimation can be readily cast into the normal form $\left(\Sigma^{\prime}\right)$. Here only $x_{1}$ is assumed accessible. Figure 2 (a) depicts the analytical expressions, that is, $x_{1}, \frac{d}{d t} x_{1}, \frac{d^{2}}{d t^{2}} x_{1}, \frac{d^{3}}{d t^{3}} x_{1}, \frac{d^{4}}{d t^{4}} x_{1}$, respectively, from top to bottom. The estimation result obtained via a high-gain observer is illustrated in Fig. 2 (b). The high-gain observer used here is expressed as 

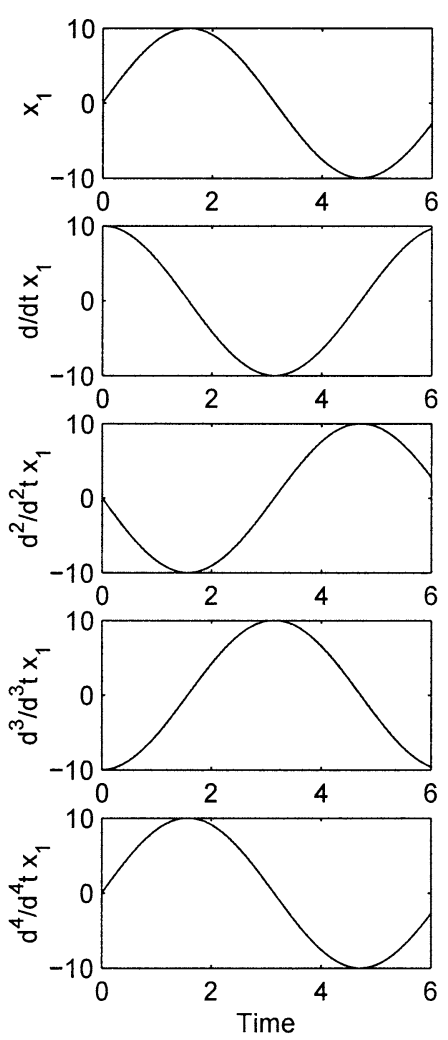

(a)
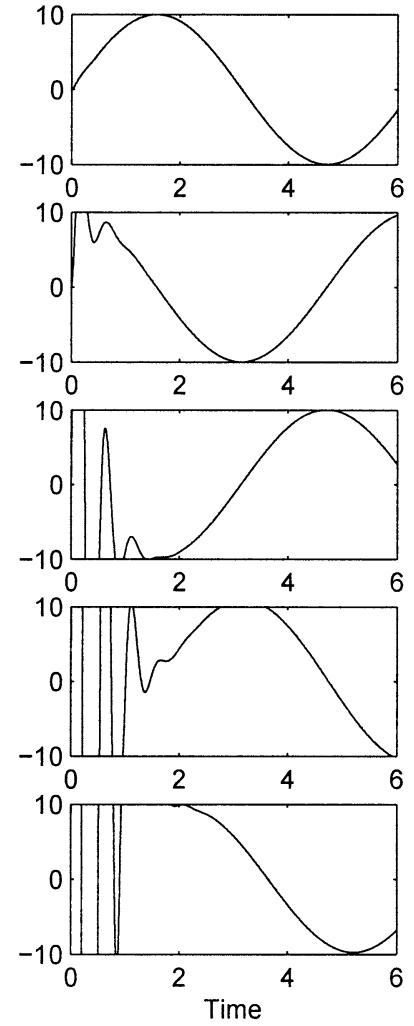

(b)
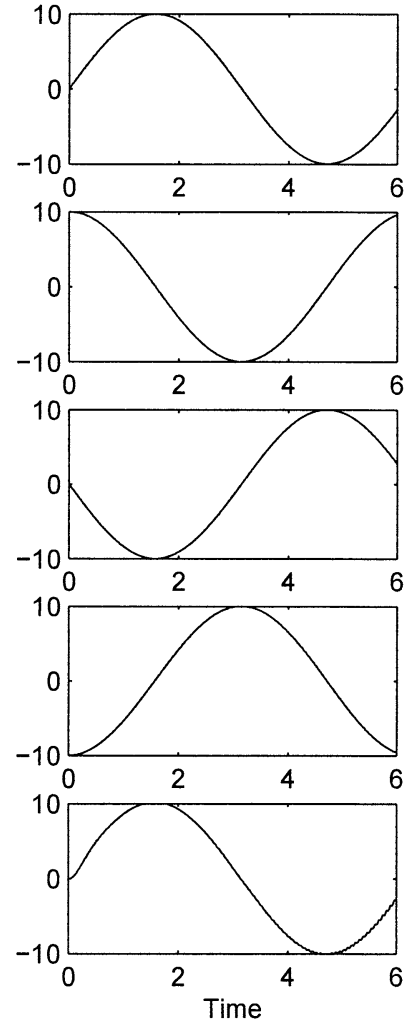

(c)

Fig. 2 The comparative results for $x_{1}=10 \sin t$ between the high-gain observer and the proposed observer. (a) analytical expressions, $x_{1}, \frac{d}{d t} x_{1}, \frac{d^{2}}{d t^{2}} x_{1}, \frac{d^{3}}{d t^{3}} x_{1}, \frac{d^{4}}{d t^{4}} x_{1}$, (b) estimation by the high-gain observer, (c) estimation by the proposed cascade observer

$$
\begin{aligned}
& \dot{\hat{x}}_{1}=\hat{x}_{2}+l_{1}\left(x_{1}-\hat{x}_{1}\right) / \epsilon \\
& \dot{\hat{x}}_{i}=\hat{x}_{i+1}+l_{i}\left(x_{1}-\hat{x}_{1}\right) / \epsilon^{i}, i=2, \cdots, 4 \\
& \dot{\hat{x}}_{5}=\quad l_{5}\left(x_{1}-\hat{x}_{1}\right) / \epsilon^{5}
\end{aligned}
$$

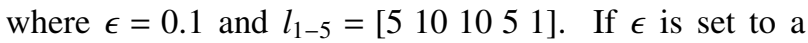
value less than 0.1 , the high-gain observer exhibits more severe peaking phenomenon ${ }^{(20)}$ than what is depicted here. In the peaking phenomenon, as the observer poles approach infinity, its exponential modes decay to zero arbitrarily fast, but the amplitudes of these modes will approach infinity thus producing an impulsive-like behavior. Figure 2 (c) shows the results via the proposed cascade observer. Design parameters of the proposed observer are $l_{1-6}=\left[\begin{array}{lllllll}130 & 100 & 80 & 40 & 10 & 5\end{array}\right]$ and $\gamma_{1-6}=\left[\begin{array}{llllll}1 & 1 & 1 & 1 & 1 & 1\end{array}\right]$. Clearly the proposed observer outperformed the high-gain observer.

\subsection{Example 2}

Consider a dynamic model for an underwater vehicle including thruster dynamics ${ }^{(24)}$ depicted in Fig. 3.

$$
\dot{x}=\left[\begin{array}{l}
\dot{x}_{1} \\
\dot{x}_{2} \\
\dot{x}_{3} \\
\dot{x}_{4}
\end{array}\right]=\left[\begin{array}{l}
x_{2} \\
u(t)-3 x_{2}\left|x_{2}\right| \\
x_{4} \\
x_{2}\left|x_{2}\right|-3 x_{4}\left|x_{4}\right|
\end{array}\right]
$$

where $x_{1}$ is the angle of the propeller, $x_{2}$ is the propeller

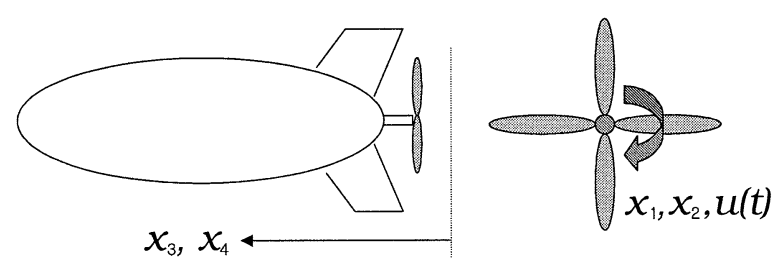

Fig. 3 Underwater vehicle

angular velocity, $x_{3}$ is the measured position, $x_{4}$ is the velocity of the vehicle, $x_{2}\left|x_{2}\right|$ represents the propeller thrust, and $u(t)$ is the torque input to the propeller. Only the propeller angle $x_{1}$ and vehicle position $x_{3}$ are measured.

The initial conditions for the system and the observer are taken as $x_{1}(0)=0, x_{2}(0)=0, x_{3}(0)=0, x_{4}(0)=5, \hat{x}_{1}(0)=$ $0, \hat{x}_{2}(0)=4, \hat{x}_{3}(0)=0$ and $\hat{x}_{4}(0)=-10$. Design parameters are set to $l_{1-6}=1000,500,10,1000,500,10$ and $\gamma_{1-6}=$ $20,20,2,20,20,2$. We set to the control input as

$$
u=\left(\begin{array}{rll}
5 & \text { for } & 0 \leq t<1 \\
-10 & \text { for } & 1 \leq t<2
\end{array}\right)
$$

Figure 4 illustrates the convergence of estimated states for the proposed cascade observer. Figure 5 shows the estimation errors of the propeller angular velocity and the vehicle velocity. 

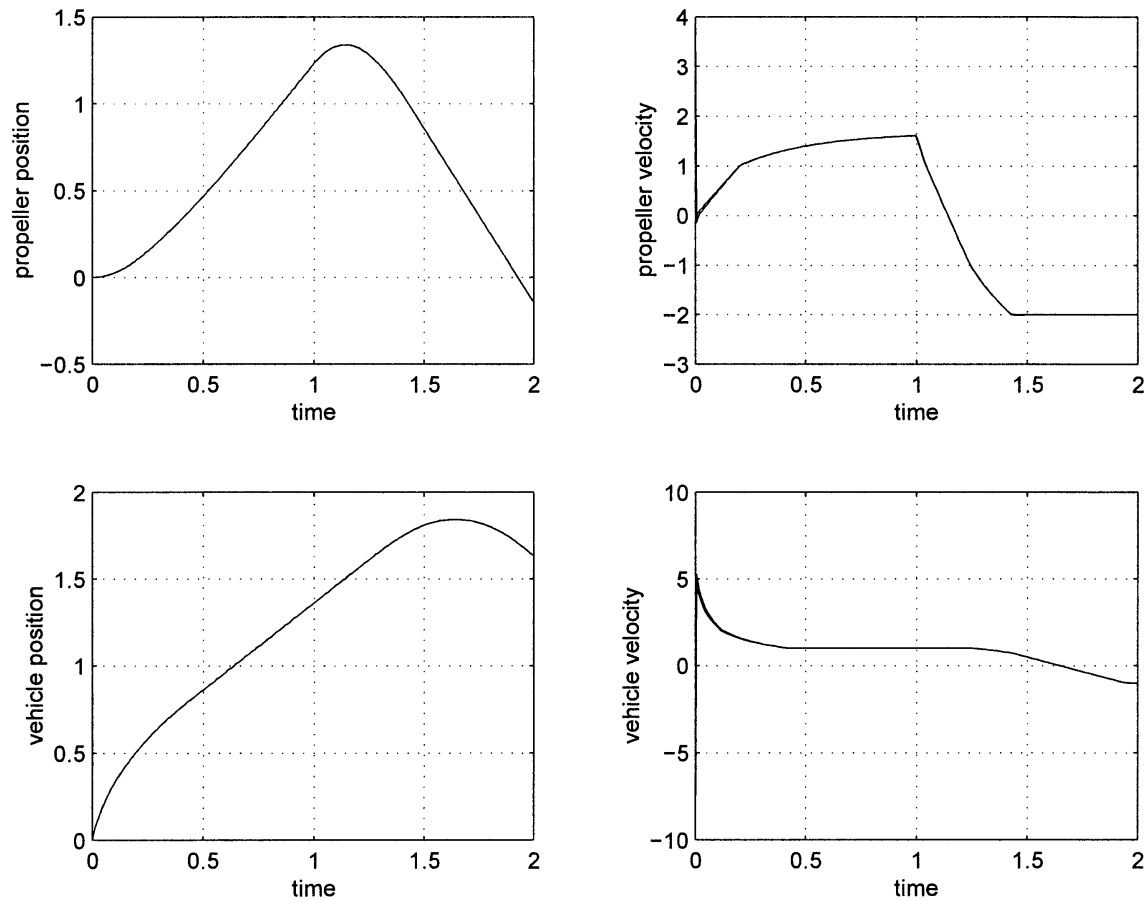

Fig. 4 The convergence of the estimated states by the proposed cascade observer
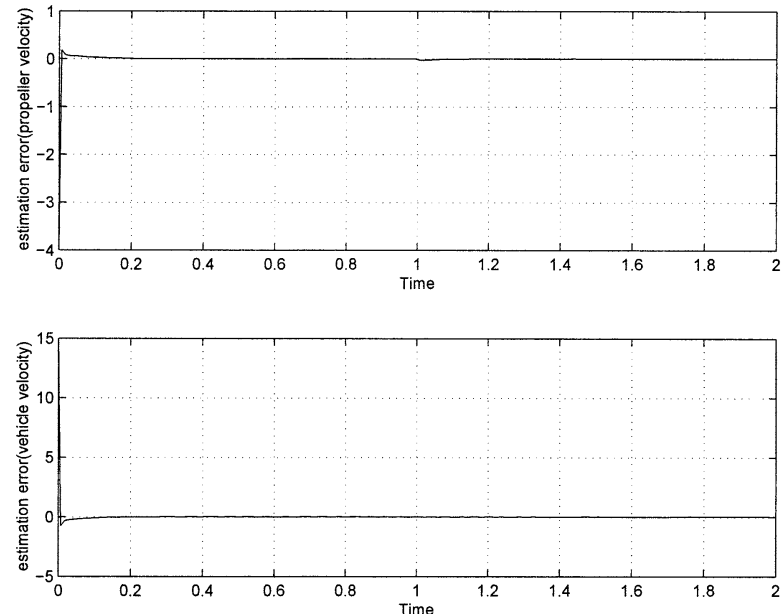

Fig. 5 The estimation errors by the proposed cascade observer

The estimation errors of the propeller angular velocity and the vehicle velocity obtained via a high-gain observer are illustrated in Fig. 6. The high-gain observer used here is expressed as

$$
\begin{aligned}
& \dot{\hat{x}}_{1}=\hat{x}_{2}+l_{1}\left(x_{1}-\hat{x}_{1}\right) / \epsilon \\
& \dot{\hat{x}}_{2}=\quad l_{2}\left(x_{1}-\hat{x}_{1}\right) / \epsilon^{2} \\
& \dot{\hat{x}}_{3}=\hat{x}_{4}+l_{3}\left(x_{3}-\hat{x}_{3}\right) / \epsilon \\
& \dot{\hat{x}}_{4}=\quad l_{4}\left(x_{3}-\hat{x}_{3}\right) / \epsilon^{2}
\end{aligned}
$$

where $\epsilon=0.01$ and $l_{1-4}=\left[\begin{array}{llll}1 & 1 & 1 & 1\end{array}\right]$.

The proposed cascade observer in Fig. 5 leads to better estimation results, compared with those of the highgain observer in Fig. 6. The proposed cascade observer also results in better performance than the metric observer
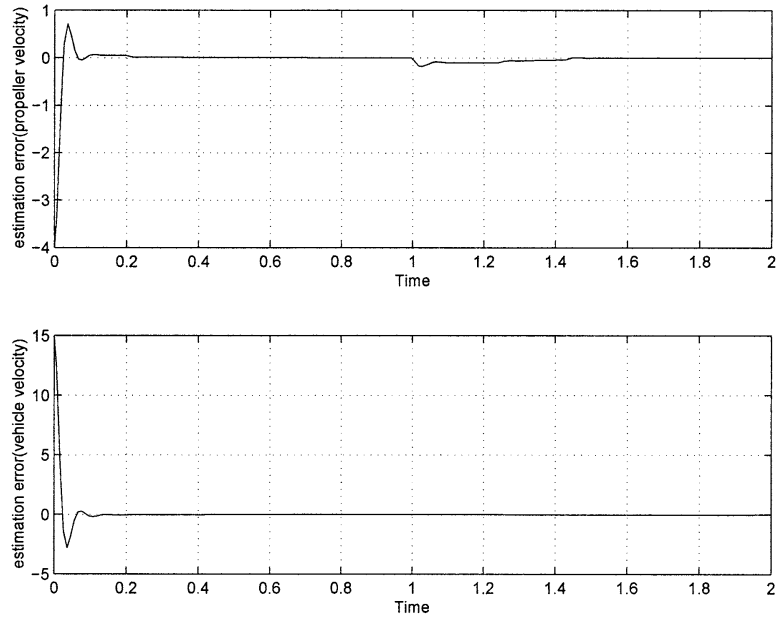

Fig. 6 The estimation errors by the high-gain observer

of Ref. (26).

Next we apply the proposed cascade observer to output feedback control of a class of SISO nonlinear systems represented by input-output models.

\section{Output Feedback Control}

\section{1 Problem statement}

Consider a SISO nonlinear system represented globally by the following $n$-th order differential equation

$$
y^{(n)}=f_{0}(\cdot)+\sum_{i=1}^{p} f_{i}(\cdot) \theta_{i}+\left(g_{0}+\sum_{i=1}^{p} g_{i} \theta_{i}\right) u^{(m)}
$$

where $u$ is the control input, $y$ is the measured output, $y^{(i)}$ denotes the $i$-th derivative of $y$, and $m<n$. $\theta$ is the vector of unknown constant parameters, and $g_{0}$ 
and $g_{i}$ are known constant parameters. The functions $f_{i}$ are known smooth nonlinearities which could depend on $y, y^{(1)}, \cdots, y^{(n-1)}, u, u^{(1)}, \cdots, u^{(m-1)}$, e.g.,

$$
f_{0}(.)=f_{0}\left(y, y^{(1)}, \cdots, y^{(n-1)}, u, u^{(1)}, \cdots, u^{(m-1)}\right) .
$$

The control objective can be described as: given a desired output $y_{r}(t)$, find a control input $u$, such that the output of the system tracks the desired trajectory with an acceptable accuracy.

We may represent system (22) in a state-space model. We extend the dynamics of the system by adding a series of $m$ integrators at the input side and represent the augmented system by a state-space model, where the states are the input, the output, and a number of their derivatives. We denote the states of these integrators by $z_{1}=u ; z_{2}=u^{(1)}$ up to $z_{m}=u^{(m-1)}$, and set $v=u^{(m)}$ as the control input of the augmented system. By taking $x_{1}=y, x_{2}=y^{(1)}$ up to $x_{n}=y^{(n-1)}$, we can represent the augmented system by the following state-space model

$$
\begin{aligned}
& \dot{x}_{i}=x_{i+1}, \quad 1 \leq i \leq n-1 \\
& \dot{x}_{n}=f_{0}(x, z)+\theta^{T} f(x, z)+\left(g_{0}+\theta^{T} g\right) v \\
& \dot{z}_{i}=z_{i+1}, \quad 1 \leq i \leq m-1 \\
& \dot{z}_{m}=v \\
& y=x_{1}
\end{aligned}
$$

where $x=\left[x_{1}, \cdots, x_{n}\right]^{T}, z=\left[z_{1}, \cdots, z_{m}\right]^{T}, f=\left[f_{1}, \cdots, f_{p}\right]^{T}$ and $g=\left[g_{1}, \cdots, g_{p}\right]^{T}$.

The initial state of the integrators are chosen such that $z(0) \in Z_{0}$, a compact subset of $R_{m}$. The change of variables

$$
\zeta_{i}=z_{i}-\frac{x_{n-m+i}}{g_{0}+\theta^{T} g}, \quad 1 \leq i \leq m
$$

transforms the last $m$ state equations of (24) into

$$
\begin{aligned}
& \dot{\zeta}_{i}=\zeta_{i+1}, \quad 1 \leq i \leq m-1 \\
& \dot{\zeta}_{m}=-\left.\frac{f_{0}(x, z)+\theta^{T} f(x, z)}{g_{0}+\theta^{T} g}\right|_{z_{i}=\zeta_{i}+x_{n-m+i} /\left(g_{0}+\theta^{T} g\right)} .
\end{aligned}
$$

Setting $x=0$ in Eq. (26) results in the zero dynamics of Eq. (24), as defined as Ref. (19).

Assumption 4 ( $i$ ) We assume that $y_{r}(t)$ is bounded and its derivatives up to $n$-th order are also bounded. $y_{r}^{(n)}(t)$ is piecewise continuous.

(ii) $g_{0}+\theta^{T} g \neq 0, \forall \theta \in \hat{\Omega}$ where the vector $\theta=$ $\left[\theta_{1}, \cdots, \theta_{p}\right]^{T}$ belongs to $\Omega$. $\hat{\Omega}$ is a convex subset of $R^{p}$ which contains $\Omega$ in its interior.

(iii) For every $\theta \in \Omega$, the system (26) has the property that for $z(0) \in Z_{0}$ and any bounded $x(t)$, the state $\zeta(t)$ is bounded.

Let us define

$$
\begin{aligned}
& Y(t)=\left[y(t), y^{(1)}(t), \cdots, y^{(n-1)}(t)\right]^{T} \\
& Y_{r}(t)=\left[y_{r}(t), y_{r}^{(1)}(t), \cdots, y_{r}^{(n-1)}(t)\right]^{T} \\
& Y_{R}(t)=\left[y_{r}(t), y_{r}^{(1)}(t), \cdots, y_{r}^{(n-1)}(t), y_{r}^{(n)}(t)\right]^{T} .
\end{aligned}
$$

We define the error vector to be

$$
e=\left[e_{1}, e_{2}, \cdots, e_{n}\right]^{T}
$$

where

$$
\begin{aligned}
& e_{1}=y-y_{r}=x_{1}-y_{r} \\
& e_{2}=\dot{y}-\dot{y}_{r}=x_{2}-\dot{y}_{r} \\
& \quad \vdots \\
& e_{n}=y^{(n-1)}-y_{r}^{(n-1)}=x_{n}-y_{r}^{(n-1)} .
\end{aligned}
$$

\subsection{Observer and controller design}

The cascade observer for output feedback control is designed as

$$
\begin{aligned}
& \dot{\hat{e}}_{1}=\hat{e}_{2}+l_{1}\left(e_{1}-\hat{e}_{1}\right)+\hat{\rho}_{1} \operatorname{sgn}\left(e_{1}-\hat{e}_{1}\right) \\
& \dot{\hat{e}}_{i}=\hat{e}_{i+1}+l_{i}\left(\dot{\hat{e}}_{i-1}-\hat{e}_{i}\right)+\hat{\rho}_{i} \operatorname{sgn}\left(\dot{\hat{e}}_{i-1}-\hat{e}_{i}\right) \quad i=2, \cdots, n \\
& \dot{\hat{e}}_{n+1}=l_{n+1}\left(\dot{\hat{e}}_{n}-\hat{e}_{n+1}\right)+\hat{\rho}_{n+1} \operatorname{sgn}\left(\dot{\hat{e}}_{n}-\hat{e}_{n+1}\right)
\end{aligned}
$$

where we denote $\left|\hat{e}_{i+1}-\ddot{\hat{e}}_{i-1}\right| \leq \rho_{i} i=1, \cdots, n$ and $\left|\ddot{\hat{e}}_{n}\right| \leq \rho_{n+1}$ (see Remark 2 of section 3.1). $\hat{\rho}_{1}, \hat{\rho}_{i}$ and $\hat{\rho}_{n+1}$ are designed according to Eq. (5) where $\hat{x}$ is replaced by $\hat{e}$.

Using a feedback linearization technique, the control input is designed as

$$
v=\frac{-K \hat{e}+y_{r}^{(n)}-\hat{f}_{0}-\hat{\theta}^{T} \hat{f}}{g_{0}+\hat{\theta}^{T} g} \equiv \psi\left(\hat{e}, z, Y_{R}, \hat{\theta}\right)
$$

where $\hat{f}_{0}=f_{0}\left(\hat{e}+Y_{r}, z\right)$ and $\hat{f}=f\left(\hat{e}+Y_{r}, z\right)$.

The parameter adaptive law is taken as

$\dot{\hat{\theta}}=\Gamma \phi$

where $\phi=2 \hat{e}^{T} P b\left[f\left(\hat{e}+Y_{r}, z\right)+g \psi\left(\hat{e}, z, Y_{R}, \hat{\theta}\right)\right]$.

Differentiating $e$ of Eq. (28) gives

$$
\begin{aligned}
\dot{e}= & A e+b\left[f_{0}\left(e+Y_{r}, z\right)+\theta^{T} f\left(e+Y_{r}, z\right)\right. \\
& \left.+\left(g_{0}+\theta^{T} g\right) v-y_{r}^{(n)}\right] \\
\dot{z}= & A_{2} z+b_{2} v
\end{aligned}
$$

where $A=\left[\begin{array}{cccccc}0 & 1 & 0 & \cdots & \cdots & 0 \\ 0 & 0 & 1 & \cdots & \cdots & 0 \\ \vdots & & & & & \vdots \\ 0 & 0 & \cdots & \cdots & 0 & 1 \\ 0 & 0 & \cdots & \cdots & 0 & 0\end{array}\right]$ and $b=\left[\begin{array}{c}0 \\ 0 \\ \vdots \\ 0 \\ 1\end{array}\right]$.

Choose a matrix $K$ such that $A_{m}=A-b K$ is Hurwits, and rewrite (32) as

$$
\begin{aligned}
\dot{e}= & A_{m} e+b\left[f_{0}\left(e+Y_{r}, z\right)+\theta^{T} f\left(e+Y_{r}, z\right)+\left(g_{0}+\theta^{T} g\right) v\right. \\
& \left.+K e-y_{r}^{(n)}\right] .
\end{aligned}
$$

Let $P=P^{T}>0$ be the solution of the Lyapunov equation

$$
P A_{m}+A_{m}^{T} P=-Q, Q=Q^{T}>0 .
$$

Theorem 3 For system (22) satisfying Assumption 2, the output feedback control problem is solvable. In particular, the output feedback control system consists of the cascade observer (29), the parameter adaptive law (31) and the control input (30).

Proof: We consider the Lyapunov function candidate

$$
V_{o}=e^{T} P e+\frac{1}{2} \tilde{\theta}^{T} \Gamma^{-1} \tilde{\theta}
$$


where $\tilde{\theta}_{i}=\theta_{i}-\hat{\theta}_{i}$.

Differentiating $V_{o}$ along the system trajectory gives

$$
\begin{aligned}
\dot{V}_{o}= & -e^{T} Q e+2 e^{T} P b\left[f_{0}+\theta^{T} f+\left(g_{0}+\theta^{T} g\right) \psi+K e\right. \\
& \left.-y_{r}^{(n)}\right]+\tilde{\theta}^{T} \Gamma^{-1} \dot{\hat{\theta}} \\
= & -e^{T} Q e+2 e^{T} P b\left[f_{0}+\theta^{T} f+\left(g_{0}+\theta^{T} g\right) \psi-\left(g_{0}\right.\right. \\
& \left.\left.+\hat{\theta}^{T} g\right) \psi+\left(g_{0}+\hat{\theta}^{T} g\right) \psi+K e-y_{r}^{(n)}\right]+\tilde{\theta}^{T} \Gamma^{-1} \hat{\hat{\theta}} \\
= & -e^{T} Q e+2 e^{T} P b\left[f_{0}+\theta^{T} f+\left(g_{0}+\theta^{T} g\right) \psi-\left(g_{0}\right.\right. \\
& \left.\left.+\hat{\theta}^{T} g\right) \psi+\left(-K \hat{e}+y_{r}^{(n)}-\hat{f}_{0}-\hat{\theta}^{T} \hat{f}\right)+K e-y_{r}^{(n)}\right] \\
& +\tilde{\theta}^{T} \Gamma^{-1} \dot{\hat{\theta}} \\
= & -e^{T} Q e+2 e^{T} P b\left[f_{0}-\hat{f}_{0}+\theta^{T} f-\hat{\theta}^{T} \hat{f}+\left(\theta^{T}-\hat{\theta}^{T}\right) g \psi\right. \\
& +K(e-\hat{e})]+\tilde{\theta}^{T} \Gamma^{-1} \hat{\hat{\theta}} \\
= & -e^{T} Q e+2 e^{T} P b\left[f_{0}-\hat{f}_{0}+\theta^{T} f+\left(\theta^{T} \hat{f}-\theta^{T} \hat{f}\right)\right. \\
& \left.-\hat{\theta}^{T} \hat{f}+\left(\theta^{T}-\hat{\theta}^{T}\right) g \psi+K(e-\hat{e})\right]+\tilde{\theta}^{T} \Gamma^{-1} \dot{\hat{\theta}} \\
= & -e^{T} Q e+2 e^{T} P b\left[f_{0}-\hat{f}_{0}+\theta^{T}(f-\hat{f})+K(e-\hat{e})\right] \\
& +\tilde{\theta}^{T} \Gamma^{-1}(\hat{\hat{\theta}}-\Gamma \phi) .
\end{aligned}
$$

Substituting Eq. (31) into Eq. (36) gives

$$
\begin{aligned}
\dot{V}_{0}= & -e^{T} Q e+2 e^{T} P b\left[f_{0}-\hat{f}_{0}+\theta^{T}(f-\hat{f})+K(e-\hat{e})\right] \\
& +2 \hat{e}^{T} P b\left[f\left(\hat{e}+Y_{r}, z\right)+g \psi\left(\hat{e}, z, Y_{R}, \hat{\theta}\right)\right]
\end{aligned}
$$

where $\tilde{e}=e-\hat{e}$.

Since $e-\hat{e}$ is converge to 0 by Theorem $1, f_{0}\left(e+Y_{r}, z\right)-$ $\hat{f}_{0}\left(\hat{e}+Y_{r}, z\right)$ and $f\left(e+Y_{r}, z\right)-\hat{f}\left(\hat{e}+Y_{r}, z\right)$ are also converge to 0 . Thus, Eq. (36) is represented by

$$
\dot{V}_{o} \leq-e^{T} Q e+\epsilon_{t}
$$

where $\epsilon_{t}$ is the term by estimation error and $\lim _{t \rightarrow \infty} \epsilon_{t}(t)=0$.

Therefore we are able to guarantee globally uniformly ultimate boundedness, that is,

$$
e(t) \rightarrow 0 \text { as } t \rightarrow \infty, \forall t \geq t_{1}
$$

for some $t_{1}>T_{1}$. The time $T_{1}$ is a function of $\epsilon_{t}$ and $T_{1}\left(\epsilon_{t}\right) \rightarrow 0$ as $\epsilon_{t} \rightarrow 0$.

\subsection{Example 3}

Consider now the problem in which the output $y$ of the nonlinear system ${ }^{(10),(21)}$

$$
\begin{aligned}
& \dot{\xi}_{1}=\xi_{2}+\theta \xi_{1}{ }^{2} \\
& \dot{\xi}_{2}=u+\xi_{3} \\
& \dot{\xi}_{3}=-\xi_{3}+y \\
& y=\xi_{1}
\end{aligned}
$$

is required to track the reference signal $y_{r}=0.1 \sin t$. The system can be represented by an input-output model

$$
y^{(3)}=(u+y-\ddot{y})+2 \theta\left(y \dot{y}+\dot{y}^{2}+y \ddot{y}\right)+\dot{u} .
$$

We set the control input $u=\int v$ where $v=\dot{u}$ in Eq. (30). Design parameters are set to $K=[243], l_{1-4}=200,1,1,1$, $\gamma_{1-4}=20,20,2,0.1, \theta=1$ and zero initial conditions for all variables except $y(0)$. Figure 7 shows the different results of tracking error for $y(0)=0.5,1.0$ and 1.5. The tracking error and observer errors for $y(0)=1.0$ are illustrated in

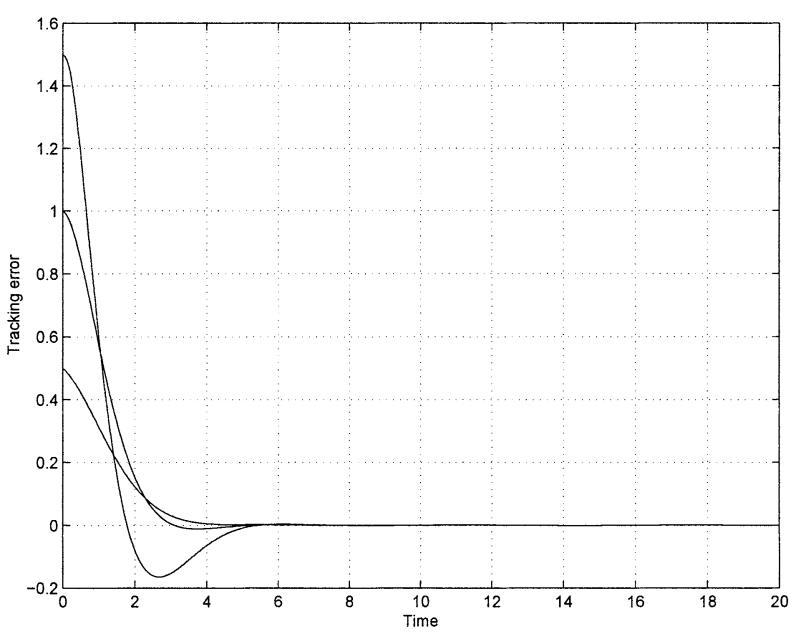

Fig. 7 Tracking error for $y(0)=0.5,1.0$ and 1.5 under $y_{r}=$ $0.1 \sin t$

Fig. 8 where observer errors 1,2 and 3 mean $e_{1}-\hat{e}_{1}, e_{2}-\hat{e}_{2}$ and $e_{3}-\hat{e}_{3}$, respectively. The control input $u$ and the control input of the extended system $v$ are also given in Fig. 9. For the sake of comparison, we have also tried to solve this problem using a high-gain observer but we were not able to come up with a working design. However, our results can be compared with a improved scheme of the highgain observer with the saturation input ${ }^{(10)}$. In Ref. (10), to avoid large control efforts during transient state, a saturation function is imposed on input. In Ref. (10) the excited control input $v$ is constrained within \pm 75 during the "peaking" of the state estimates, whereas the proposed method in this paper utilizes less control efforts $(-18<v<5)$ as shown in Fig. 9 and achieves better performance. Indeed our results are comparable to those in Ref. (21) using fullstate feedback. Figure 10 shows simulation results for $y_{r}(t)=0.5$. Please note that this case violates the persistently exciting condition in Ref. (10). Design parameters are chosen to be the same as the case of $y_{r}=0.1 \sin t$.

\section{Conclusion}

In this paper, we present a new observer, namely a cascade observer, for a general class of nonlinear SISO systems. The proposed observer features a cascade structure and adaptive observer gains. In doing so the cascade observer attempts to overcome some of the typical problems that may pose to a high-gain observer. As in the case of a high-gain observer, the cascade observer structure is simple and universal in the sense that it is independent of the system dynamics and parameters. It is shown that the estimation errors converge to zero and all the internal variables are globally uniformly bounded.

Based on the cascade observer, an adaptive output feedback controller is designed for a class of SISO nonlinear system represented globally by a $n$ th-order differential equation. The output feedback controller has the same structure as a state feedback controller. Glob- 



Fig. 8 Tracking error and observer errors for $y(0)=1.0$ under $y_{r}=0.1 \sin t$
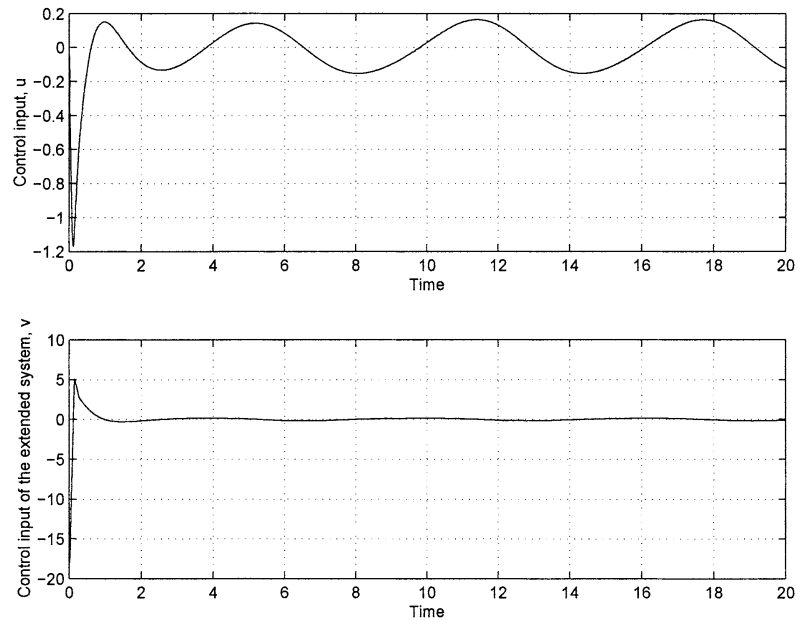

Fig. 9 Control input $u$ and control input of the extended system $v$ for $y(0)=1.0$ under $y_{r}=0.1 \sin t$

ally uniformly ultimate boundedness of tracking errors is achieved for the nonlinear systems with unknown parameters. The proposed adaptive output feedback controller does not use filtered transformations ${ }^{(22),(23)}$. Moreover, in our design, conditions such as global growth conditions on the nonlinearities (as in Ref. (16)) and the persistently exciting condition (as in Refs. (10) and (16)) are not required for tracking error convergence. Research is ongoing to address robustness issues for the proposed observer and controller in the presence of bounded disturbances and uncertainties.

\section{Acknowledgment}

This work was supported by the Post-doctoral Fel-
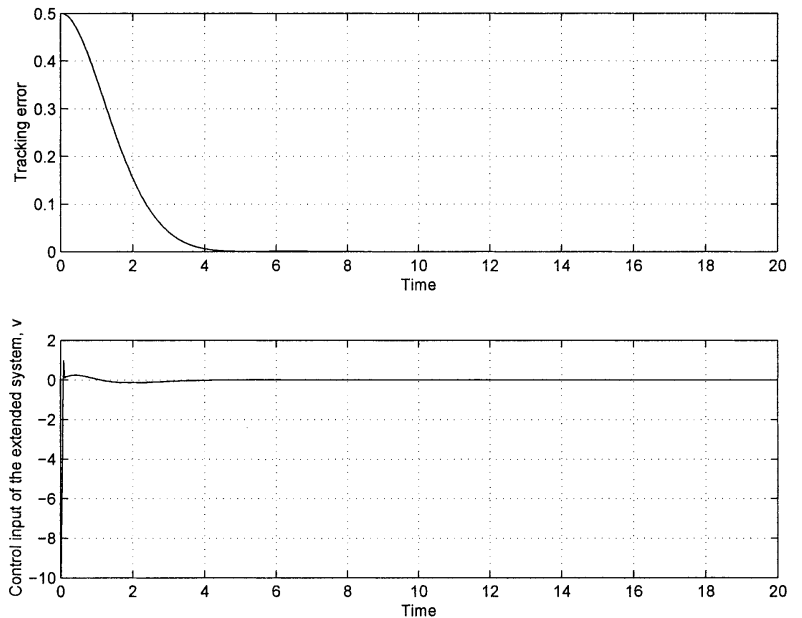

Fig. 10 Tracking error and control input of the extended system $v$ for $y(0)=1.0$ under $y_{r}=0.5$

lowship Program of Korea Science \& Engineering Foundation (KOSEF).

\section{References}

( 1 ) Ioannou, P.A. and Sun, J., Robust Adaptive Control, (1996), Prentice Hall.

( 2 ) Ahmed-Ali, T. and Lamnabhi-Lagarrige, F., Sliding Observer-Controller Design for Uncertain Triangular Nonlinear Systems, IEEE Trans. on Automatic Control, Vol.44 (1999), pp.1244-1249.

( 3 ) Misawa, E.A. and Hedrick, J.K., Nonlinear Observers: A State-of-the-Art Survey, Trans. ASME, J. Dynamic Syst., Measurement and Contr., Vol.111 (1989), pp.344-352.

( 4 ) Slotine, J.J., Hedrick, J.K. and Misawa, E.A., On Slid- 
ing Observers for Nonlinear Systems, Trans. ASME, J. Dynamic Syst., Measurement and Contr., Vol.109 (1987), pp.245-252.

( 5 ) Wang, G.B., Peng, S.S. and Huang, H.P., A Sliding Observer for Nonlinear Process Control, Chemical Engineering Science, Vol.52 (1997), pp.787-805.

( 6 ) Besancon, G., Further Results on High Gain Observers for Nonlinear Systems, Proceedings of the 38th IEEE Conference on Decision and Control, Vol.3 (1999), pp.2904-2909.

( 7 ) Shin, E.S. and Lee, K.W., Robust Output Feedback Control of Robot Manipulators Using High-Gain Observer, Proceedings of the IEEE International Conference on Control Applications, No.1 (1999), pp.881886.

( 8 ) Dabroom, A. and Khalil, H.K., Discrete-Time Implementation of High-Gain Observers for Numerical Differentiation, International Journal of Control, Vol.72 (1999), pp.1523-1537.

(9) Isidori, A., Nonlinear Control Systems, (1995), Springer-Verlag.

(10) Khalil, H.K., Adaptive Output Feedback Control of Nonlinear Systems Represented by Input-Output Models, IEEE Trans. Automat. Control, Vol.41 (1996), pp.177-188.

(11) Martinez-Guerra, R., Garrido, R. and Osorio-Miron, A., Parametric and State Estimation by Means of HighGain Nonlinear Observers: Application to a Bioreactor, American Control Conference, (2001), pp.3807-3808.

(12) Zhang, Q. and Xu, A., Implicit Adaptive Observers for a Class of Nonlinear Systems, American Control Conference, (2001), pp.1551-1556.

(13) Hammouri, H. and Marchand, N., High Gain Observer for a Class of Implicit Systems, Proceedings of the 39th IEEE Conference on Decision and Control, (2000), pp.804-808.

(14) Aizerman, M.A. and Piatnitskiy, Y.S., Theory of Dynamic Systems Which Incorporate Elements with Incomplete Information and Its Relation to the Theory of Discontinuous Systems, Journal of the Franklin Institute, Vol.306 (1978).

(15) Heredia, J.A. and Yu, W., A High-Gain ObserverBased PD Control for Robot Manipulator, American Control Conference, (2000), pp.2518-2522.

(16) Jankovic, M., Adaptive Nonlinear Output Feedback Tracking with a Reduced Order High-Gain Observer, American Control Conference, (1994), pp.875-879.

(17) Tornambe, A., High-Gain Observers for Nonlinear Systems, Int. J. of Systems Science, (1992), pp.14751489.

(18) Fliess, M., Generalized Controller Canonical Form for Linear and Nonlinear Dynamics, IEEE Trans. Automat. Contr., Vol.35 (1990), pp.994-1001.

(19) Slotine, J.E. and Li, W., Applied Nonlinear Control, (1991), Prentice Hall.

(20) Esfandiari, F. and Khalil, H.K., Output Feedback Stabilization of Fully Linearizable Systems, International Journal of Control, Vol.56 (1992), pp.1007-1037.
(21) Kanellakopoulos, I., Kokotovic, P.V. and Morse, A.S., Systematic Design of Adaptive Controllers for Feedback Linearizable Systems, IEEE Trans. Automat. Control, Vol.36 (1991), pp.1241-1253.

(22) Marino, R. and Tomei, P., Global Adaptive OutputFeedback Control of Nonliner Systems, Part I: Linear Parameterization, IEEE Trans. Automat. Control, Vol.38 (1993), pp.17-32.

(23) Jankovic, M., Adaptive Nonlinear Output Feedback Tracking with a Reduced Order High-Gain Observer, Proceedings of the American Control Conference, (1994), pp.875-879.

(24) Yoerger, D., Cooke, J. and Slotine, J.E., The Influence of Thruster Dynamics on Underwater Vehicle Behavior and Their Incorporation into Control System Design, IEEE Trans. Oceanic Engineering, Vol.15 (1990), pp.167-178.

(25) Arfken, G., Mathematical Methods for Physicists, (1985), pp.527-529, Academic Press.

(26) Lohmiller, W. and Slotine, J.E., Applications of Metric Observers for Nonlinear Systems, IEEE Int. Conf. Control Applications, (1996), pp.367-372.

(27) Tornambe, A., Use of Asymptotic Observer HavingHigh-Gains in the State and Parameter Estimation, Proceedings of the 28th IEEE Conference on Decision and Control, (1989), pp.1791-1794.

(28) Bullinger, E. and Allgower, F., An Adaptive HighGain Observer for Nonlinear Systems, Proceedings of the 36th IEEE Conference on Decision and Control, (1997), pp.4348-4353.

(29) Tornambe, A., High-Gain Observers for Nonlinear Systems, Int. J. of Systems Science, (1992), pp.14751489.

(30) Belanger, P.R., Estimation of Angular Velocity and Acceleration from Shaft Encoder Measurements, IEEE Conference on Robotics and Automation, (1992), pp.585-592.

(31) Canudas de Wit, C. and Fixot, N., Robot Control via Robust State Estimated Feedback, IEEE Trans. on Automatic Control, Vol.36 (1991), pp.1497-1501.

(32) Klafter, R.D., Chmielewski, T.A. and Negin, M., Robotic Engineering - An Integrated Approach, (1989), Prentice Hall, Englewood Cliffs, NJ.

(33) Sussmann, H.J., Single Input Observability of Continuous Time Systems, Math Systems Theory, Vol.12 (1979), pp.371-393.

(34) Gauthier, J.P. and Bornard, G., Observability for Any $u(t)$ of a Class of Bilinear Systems, IEEE Trans. on Automatic Control, Vol.26 (1981), pp.922-926.

(35) Esfandiari, F. and Khalil, H.K., Output Feedback Stabilization of Fully Linearizable Systems, Int. J. of Control, Vol.56 (1992), pp.1007-1037.

(36) Filippov, A.F., Differential Equations with Discontinuous Right Hand Sides, Mathematicheskii Sbornek, (in Russian), Vol.5 (1960), pp.99-127, English Translation, Amer. Math. Soc. Tran., Vol.42 (1964), pp.199231. 